\title{
Evidence against signal enhancement as a mechanism of direct selection by color
}

\author{
EsTHer VierCK AND JeFF MILler \\ University of Otago, Dunedin, New Zealand
}

\begin{abstract}
Two of the possible mechanisms that have been put forward to explain precuing effects are signal enhancement and uncertainty reduction. Signal enhancement leads to processing advantages for valid information because the signal at the known input channel is enhanced, whereas uncertainty reduction allows observers to ignore confusing distractor items in a display. Both mechanisms have been reported to be involved in location precuing, but it is still unclear which of these two mechanisms is responsible for color cuing effects. Two experiments are reported in which expectancy for a certain color in a single-item display was created. Targets were presented briefly and were masked. If color cues produce signal enhancement, then a color cuing effect should result. If color cues only allow uncertainty reduction, however, there should be no color cuing effect because there are no distractors in single-item displays. The results of both experiments favor uncertainty reduction as the mechanism behind color cuing, because no signs of signal enhancement-based cuing effects were observed.
\end{abstract}

Providing advance location information in multipleitem displays has frequently been shown to lead to processing advantages (see, e.g., Posner, Snyder, \& Davidson, 1980; Theeuwes, 1989). These benefits are reported for various tasks, including detection (Posner et al., 1980), discrimination (Theeuwes, 1989), and identification (Cheal \& Gregory, 1997). Typically, the effects of advance information are studied in precuing tasks. As an example of such a task, McCann, Folk, and Johnston (1992) cued one of two locations in a lexical decision task. Cues could be either valid or invalid. The authors found that responses were faster for both words and nonwords displayed at the cued location than for those at the uncued location. In addition, accuracy was higher in these valid-cue trials than in invalid trials. This and many analogous results clearly demonstrate an advantage of advance knowledge of stimulus location.

Within theories of attention, two mechanisms that can explain this benefit have been put forward. The first one is often referred to as signal enhancement and is based on the assumption of capacity limitation within the human information processing system (e.g., Bashinski \& Bacharach, 1980). Because there are only limited resources available, it is necessary to have a mechanism allocating resources where they are needed to ensure fast and effective processing of relevant information. Attention is regarded as the process responsible for such allocation. According to this view of attention, a location precue allows participants to allocate a larger share of attention to the cued location in a top-down manner, with the exact share determined by cue validity. Consequently, more processing resources are assigned to this known input channel, enhancing or prioritizing the processing of its signal and ultimately leading to faster and more accurate processing. Invalid cues direct participants to allocate the majority of attentional resources to the wrong channel, leading, therefore, to processing costs. In the neutral cue condition, in which no advance location information is available, processing resources are simply distributed evenly over the whole display.

One other mechanism that can account for the results of location precuing experiments with multiple items is uncertainty reduction (sometimes also referred to as noise reduction; Shiu \& Pashler, 1994). According to this view, precuing benefits can be conceived of as a statistical phenomenon (e.g., Kinchla, Chen, \& Evert, 1995). When distractor items are present in the field, participants may incorrectly confuse one of these with a target, because of perceptual noise. The response to a distractor item will necessarily be at chance accuracy, so this perceptual noise degrades performance. A location precue allows the observer to give extra weight to the perceptual information from the location likely to contain the target (cf. Kinchla et al., 1995), reducing the likelihood of confusions caused by distractor items in unlikely locations, and it therefore leads to a performance benefit in valid trials. In invalid trials, however, more mistakes and slower responses are likely, because extra weight is given to the distractor item at the cued location. In neutral trials, all locations are weighted equally, so performance should be worse than that of valid trials but better than that of invalid cue trials.

To explore which of these two possibilities better explains the results, multiple-item displays are unsuitable, because both mechanisms would predict an advantage of cuing in this situation. For that reason, attempts to investigate which of these mechanisms is responsible for the

E.Vierck, esther@psy.otago.ac.nz 
observed precuing benefits have concentrated on advance location information provided in single-item rather than multiple-item displays. If signal enhancement is responsible for the cuing advantage, then a cuing benefit should remain in a situation where only one stimulus is presented at one of several possible locations, because allocating attention to the correct location should enhance processing of the signal presented in that location. If, on the other hand, the location cuing benefit is due to uncertainty reduction, then no cuing effect should be observed in a situation with only one stimulus in the display, because there are no confusing distractor items present.

Studies with single-item displays have produced mixed results, with evidence found in favor of signal enhancement, uncertainty reduction, or both (e.g., Bashinski \& Bacharach, 1980; Henderson, 1991; Van der Heijden, Schreuder, \& Wolters, 1985). For example, Bashinski and Bacharach asked participants to detect a target in a precuing design with two possible target locations. Cues were valid, neutral, or invalid, and targets were presented very briefly and only in half of the trials. This design enabled the authors to use a signal-detection procedure to analyze the data. They found that the visual sensitivity at cued locations was enhanced in comparison with the sensitivity at uncued locations. Although these results seem to suggest signal enhancement, Shiu and Pashler (1994) pointed out that uncertainty reduction can also affect $d^{\prime}$, especially because the reduction of noise will lower the false alarm rate. Henderson (1991) also investigated location precue benefits in brief and masked displays and reported a large benefit in accuracy for the valid cue condition. Other researchers found no advantage with advance location information in single-item displays (e.g., Grindley \& Townsend, 1968; Palmer, Ames, \& Lindsey, 1993; Shiu \& Pashler, 1994). Indeed, Shiu and Pashler suggested that earlier evidence for cuing effects in single-item displays (e.g., Henderson, 1991) may have depended on the use of multiple masks, which could allow confusions to occur even at locations where no stimulus had been presented. Yet, other investigators have reported signs of both signal enhancement and uncertainty reduction in their experiments (Cheal \& Gregory, 1997; Henderson, 1996; Lu \& Dosher, 2000; Luck, Hillyard, Mouloua, \& Hawkins, 1996). The results of these studies have also suggested that a cuing interval of at least $200-250 \mathrm{msec}$ is needed to obtain location cuing effects in single-item displays. Based on the full set of studies, however, a fair summary seems to be that both signal enhancement and uncertainty reduction contribute to location precuing benefits.

The studies reviewed so far were concerned only with processing advantages observed in location cuing paradigms. However, cuing benefits have also been observed for other features - in particular, color (e.g., Brawn \& Snowden, 1999) - and the analogous question concerning signal enhancement versus uncertainty reduction is also relevant for these features, because both mechanisms are able to explain color cuing effects in multiple-item displays. This article presents two experiments investigating signal enhancement versus uncertainty reduction as accounts of the effects of color cuing.
Previous studies have not attempted to determine whether signal enhancement or uncertainty reduction underlies color cuing benefits, mainly because the benefits observed through advance color information have only recently been attributed to color as a means for direct selection. Previously, color was regarded mostly as a means to guide attention to the location of the cued item where selection then took place (e.g., Cave \& Pashler, 1995; Garner, 1987; Tsal \& Lamy, 2000; Tsal \& Lavie, 1988). Some researchers did provide indirect evidence against signal enhancement while examining the attentional guidance functions of color (Brawn \& Snowden, 1999; Moore \& Egeth, 1998), but the question of uncertainty reduction has not been explicitly addressed.

For example, Brawn and Snowden (1999) used a multiple-item display in which half of the items were red and the other half were green. In each trial, one of these items would change luminance, and participants were asked to discriminate the direction of this change. In some trials, valid information about the target color was provided. Responses in these trials were significantly faster than responses in trials without color information. In a subsequent experiment, the intensity of the luminance change was varied. Brawn and Snowden argued that if informative color information leads to direct selection, then the effect of color cuing on reaction time (RT) should not depend on the intensity of the flash, because attention would already be focused on the correct item. They found, however, that the cuing effect did vary with the intensity of the flash, which they interpreted as a sign that color affected the selection process before attention reached the target. They concluded, accordingly, that advance color information does not enhance the signal of the target item and that the function of color is that of guidance to a cued location.

A study by Moore and Egeth (1998) also explored the role of color in selection by testing for signal enhancement. Participants had to detect a digit among several letters. Approximately half of the items in the display were blue; the remaining items were green. Participants received prior information about the likelihood of the target digit being in one of these two colors. Evidence of an effect of color knowledge was found, because responses were faster when the target appeared in the color with higher probability. In this experiment, the display remained on the screen until response execution. In a further experiment with brief, masked displays, however, no benefit of advance color information was found. Accordingly, the authors concluded that color information does not lead to an enhancement of the sensory quality of the target, because in this case, a benefit should have been present even when the processing time of the target was restricted. Like Brawn and Snowden (1999), they concluded that color guides attention to the target location where selection then takes place, but that direct selection by color is not possible without mediation by location.

A recent experiment by Vierck and Miller (2005) showed, however, that direct selection by color can take place. They used a rapid serial visual presentation (RSVP) task. In this paradigm, all items are presented at fixation. 
Consequently, selection has to take place in time rather than in space. Without any variation in location, any observed color cuing benefits must be due to direct selection by color rather than color selection mediated by location. Vierck and Miller (2005) presented 15 colored letters in their upper- or lowercase forms for $70 \mathrm{msec}$ each. Participants were asked to determine whether a prespecified target letter was presented in its upper- or lowercase version. At the beginning of each trial, the color of the target letter was cued, and the cue could be valid, neutral, or invalid. Although this color information was not essential to performing the task, the validity of the color cue did have an effect on performance, with valid color cues leading to better performance and invalid color cues leading to worse performance as compared with the neutral cue condition. These results indicate that color can be used for direct selection of relevant items without mediation by location.

The new results of Vierck and Miller (2005) suggest that previously reported color cuing benefits, which have generally been interpreted wholly in terms of selection mediated by location, might have been at least partially due to direct selection by color. Consequently, the findings of Brawn and Snowden (1999) and Moore and Egeth (1998) might also reflect direct selection by color to some degree. However, because these findings stem from a design in which location varies, a clear decision against signal enhancement in direct selection by color cannot be made. One cannot exclude that color functioned in these designs as a guide to a certain location. Location information has been shown to be highly effective in selection (e.g., Tsal \& Lavie, 1993). It is therefore conceivable that location is the dominant feature in attentional selection in any design, including potentially different target positions. An accurate assessment regarding signal enhancement as the mechanism behind color cuing benefits is only possible in a single-item display where location variation cannot contribute.

To investigate directly whether signal enhancement is involved in the observed direct selection by color (Vierck $\&$ Miller, 2005), the present experiments used single-item displays. In Experiment 1, a color cue was provided in a four-choice discrimination task, whereas Experiment 2 created color expectations through varying stimulus color probabilities. Both experiments displayed only one item per trial, followed by a mask. If color cuing leads to signal enhancement, then discrimination accuracy should be greater following valid cues than following invalid ones. If color cuing leads only to uncertainty reduction, however, cue validity should have no effect in such single-item displays because of the absence of distractor items.

\section{EXPERIMENT 1}

In the first experiment, participants were asked to perform a discrimination task. In each trial, participants had to identify which one of four possible geometrical forms was presented. Forms were presented in either red or green. The most likely color was cued, then the form was presented very briefly and was followed by a mask. Identical to location cuing experiments conducted with single-item displays, the color cue provided only additional informa- tion, and it was possible to perform the discrimination task correctly without processing the cue at all. Thus, in order to ensure cue processing, participants were also asked to report back the cued color at the end of each trial.

Cue presentation was very long to ensure that there was enough time to allow a potential signal enhancement to occur (Luck et al., 1996). In contrast to location cuing paradigms, the possibility of eye movements was not a confounding factor here, because all stimuli were presented at fixation.

If color cuing directly enhances the perceptual quality of a cued item, then providing valid color information should lead to a processing advantage. On the other hand, if color cuing only allows the reduction of uncertainty by eliminating confusing information, then color cuing should not be beneficial when there is only one item in the display, because there is no uncertainty about which item should be selected.

\section{Method}

Participants. The participants were 20 undergraduate students ( 3 male) at the University of Otago, New Zealand. Their ages ranged from 17 to 21 years. Students participated as part of a first-year course requirement. All participants had normal color vision.

Stimuli. The stimuli were four geometrical forms: triangle, square, pentagon, and hexagon. The triangle was presented upside down with its base nearest the top of the screen. Its two equal sides were $1.2^{\circ}$ visual angle, and the base had a length of $1.6^{\circ}$. The square had a side length of $1.1^{\circ}$. For the pentagon, base length was $0.9^{\circ}$, and its height was $1.1^{\circ}$; the sides were $0.7^{\circ}$ long. Lastly, the hexagon was $1.1^{\circ} \mathrm{high}$; the bottom and the top line were $0.6^{\circ}$ long, whereas the four remaining sides were each $0.7^{\circ}$ long. Two colors were used; these were red and green, with the luminance of these two colors matched as described below. Each stimulus was followed by a mask that had the form of a $2.8^{\circ} \times 2.3^{\circ}$ square and was filled for each trial differently with random lines in green (CIE $x, y$ chromaticity coordinates of .268/.612) and red (CIE $x, y$ chromaticity coordinates of .589/.359). The color of the mask was brighter than the color of the target in order to ensure that masking was effective.

Precues consisted of the letter " $X$," which was displayed either in the same color as the forthcoming target stimulus (valid trials) or in a different color (invalid trials). The " $\mathrm{X}$ " was $1.2^{\circ}$ wide and $1.1^{\circ}$ high. The stimuli were presented on an IBM PC-compatible computer and viewed from a distance of approximately $59 \mathrm{~cm}$.

All stimuli were presented one at a time in the center of the screen on a dark background. Participants were required to respond with their index and middle fingers to indicate the geometrical form of the target. For right hand responses, the index finger pressed the comma (“,") key, and the middle finger pressed the period (“.”) key; for left hand responses, the index finger pressed the "c" key, and the middle finger pressed the " $\mathrm{x}$ " key on a standard computer keyboard. The assignment of the four geometrical forms to the response keys was randomized across participants. As a reminder of the formresponse key assignment, small tiles with drawings of these forms were attached at the bottom part of the screen. At the end of each trial, participants were asked to indicate the color of the precue. In the case of a green "X," they were asked to press the " $z$ " key, and in the case of a red "X," they were asked to press the slash ("“") key.

Procedure. The experiment consisted of 11 blocks: 1 practice block with 28 trials, and 10 experimental blocks with 40 trials each. For both block types, the presentation of trials was randomized. A single session was approximately 50 min long.

To ensure that a possible difference between the two colors used in this experiment would be due to color (hue) rather than brightness, a procedure was implemented to equate the luminance of the red and green stimuli. Each participant was asked to run through a 
short program before the actual experiment. In this program, the screen was divided into red and green colored fields. Each field had the shape of a rectangle with $13.1^{\circ}$ width and $18.5^{\circ}$ length. The red field was stable in its luminance (CIE $x, y$ chromaticity coordinates of $.590 / .358$, luminance $1.41 \mathrm{~cd} / \mathrm{m}^{2}$ ). Participants adjusted the green field to achieve subjectively equal luminance by pressing the quotation mark (")") key to make the green dimmer and the slash ("/") key to make the green brighter. The luminance of the different greens ranged across participants from $0.47 \mathrm{~cd} / \mathrm{m}^{2}$ to $2.7 \mathrm{~cd} / \mathrm{m}^{2}$. Each participant's individually matched value for green was then integrated in the main experiment. These somewhat low luminances were chosen to ensure effective masking, because brighter stimuli could be seen relatively easily despite masking.

The practice block was designed to make participants familiar with the particular stimulus-response mapping. In the practice block, each trial started with a plus sign, which served as a warning signal and a fixation point for the participant. The plus sign was presented for $300 \mathrm{msec}$ and was followed $500 \mathrm{msec}$ later by the precue, which remained on the screen for $1,500 \mathrm{msec}$. The target stimulus was presented $500 \mathrm{msec}$ after the offset of the precue. It remained for $300 \mathrm{msec}$ and was immediately followed by the mask. During practice, the stimuli were presented in white, as was the warning cross and the precue. The mask remained visible until a response was initiated. Each form was presented seven times per block in a random fashion, making a total of 28 trials in the practice block.

The participants received new verbal instructions for the subsequent experimental blocks. These blocks were the same as the practice blocks, with the following exceptions: Forms and precues were presented in either red or green. Both colors appeared equally often. The cue was valid in $80 \%$ of the trials (i.e., the cue and form had the same color) and invalid in $20 \%$ of the trials (i.e., the cue and form had different colors). In contrast to the practice block, the presentation of the target stimulus in experimental blocks was much briefer. In the first experimental block, the target was presented for $60 \mathrm{msec}$. In the blocks that followed, the presentation time was adjusted according to the accuracy rate of the participant in the previous block. If accuracy was higher than $80 \%$ in the previous block and the presentation time was above a base level of $40 \mathrm{msec}$, it was decreased by $10 \mathrm{msec}$ for the next block; if the presentation time was already under base level, then the reduction was only $5 \mathrm{msec}$. The reduction was also $5 \mathrm{msec}$ when accuracy was between $60 \%$ and $80 \%$. Presentation time remained the same when accuracy was in the range of $40 \%$ to $60 \%$, and it was increased by $5 \mathrm{msec}$ when accuracy was below $40 \%$. Presentation times could be adjusted in 5-msec steps via the FASTVGA routine (Spitczok von Brisinski, 1994).

Participants were instructed to make the form identification response immediately after the discrimination of the target stimulus. After this response was made, a question took the place of the mask, asking the participant to indicate the color of the precue. The latter question was included to ensure that participants would attend to the cue. Following an incorrect response in the discrimination task, acoustic feedback was provided in the form of a $400-\mathrm{Hz}$ tone for $100 \mathrm{msec}$. In cases of errors in operation - for example, using the wrong response keys or responding before the target was shown-a visual reminder was displayed on the screen. The next trial began after approximately $1,000 \mathrm{msec}$.

\section{Results and Discussion}

Trials with RTs shorter than $200 \mathrm{msec}$ (approximately $0.12 \%$ of all trials) were excluded from the analysis of percentage correct (PC). The aim of this cut-off value was to exclude responses that were based on anticipations. For analysis of RT, there was also an upper cut-off value of 3,000 msec. In total, $3.69 \%$ of all trials exceeded this value and were therefore excluded. Table 1 displays mean accuracies and RTs for valid and invalid trials in both trial conditions.
Analysis of accuracy. An ANOVA was conducted using the within-subjects factors of validity (valid vs. invalid), cue type (green vs. red), and form (triangle, square, pentagon, hexagon). Where appropriate, GreenhouseGeisser corrections (Huynh, 1978) were performed, and adjusted $p$ values are reported.

The main effect of color cue validity was not significant $\left[F(1,19)=0.24, M S_{\mathrm{e}}=87.22, p=.63\right]$. To estimate the population color cuing effect, we calculated a $95 \%$ confidence interval. For this alpha level, the effect size lies between $-2.70 \%$ and $1.68 \%$. Thus, although the null hypothesis of no effect cannot be proved to be exactly true, the results do demonstrate that there is at most a very small effect of color cuing under these conditions.

The interaction between validity and precue color was significant $\left[F(1,19)=5.02, M S_{\mathrm{e}}=1,041.8, p<.05\right]$, with responses to red targets (i.e., valid trials with red precue, invalid trials with green precue) being more accurate (average of $60.9 \%$ ) and responses to green targets (i.e., valid trials with green precue, invalid trials with red precue) being less accurate (average of $52.85 \%$ ). These results indicate an advantage for red targets despite the fact that colors were matched in luminance. In addition, there was a main effect of stimulus form $[F(3,57)=11.90$, $\left.M S_{\mathrm{e}}=650.48, p<.001\right]$, with triangles being more accurately discriminated than the other forms.

Analysis of RT. For the analysis of RT, the main effect of validity was again not significant $[F(1,19)=2.76$, $\left.M S_{\mathrm{e}}=8,307.8, p=.113\right]$. As for accuracy, a 95\% confidence interval was calculated to estimate the population color cuing effect, which was between -4.4 msec and $38.2 \mathrm{msec}$. Again, a null effect is possible, and the range shows that the maximum possible color cuing effect is less than $40 \mathrm{msec}$. The main effect for precue colors was significant $\left[F(1,19)=7.38, M S_{\mathrm{e}}=12,365.8, p<.02\right]$, with responses following red cues being faster than those following green cues. There was also a three-way interaction between validity, cue type, and form $[F(3,57)=4.68$, $\left.M S_{\mathrm{e}}=10,526.8, p<.01\right]$. When the cue was red, performance was only slightly affected by validity, whereas when the cue was green, performance for invalid trials was better than that for valid trials. This result reflects the advantage found for red stimuli in the analysis for accuracy. It seems that whenever the color red was part of the trial, either as a precue or as a target, RTs were faster in comparison with trials that consisted of only green stimuli. This effect was further mediated by form, with triangles showing a par-

Table 1

Average Percentage Correct (PC) and Reaction Time (RT, in Milliseconds) As a Function of Cue Color and Validity in Experiment 1

\begin{tabular}{ccc}
\hline & \multicolumn{2}{c}{ Dependent Variable } \\
\cline { 2 - 3 } Trial Condition & PC & RT \\
\hline Valid & 52.3 & 1,233 \\
Green cue & 61.0 & 1,173 \\
$\quad$ Red cue & & \\
Invalid & 60.8 & 1,188 \\
Green cue & 53.4 & 1,183 \\
Red cue &
\end{tabular}


ticularly strong advantage for red stimuli. The main effect of forms was also significant $\left[F(3,57)=11.69, M S_{\mathrm{e}}=\right.$ $62,265.0, p<.001]$, confirming a strong advantage for triangles in comparison with the other forms. ${ }^{1}$

In summary, we found no validity effect in this experiment with single-item displays, either in accuracy or in RT. These results can therefore not be regarded as support for signal enhancement via color cues.

\section{EXPERIMENT 2}

The absence of any cuing effect in the previous experiment argues against signal enhancement as the mechanism responsible for color cuing effects, but alternative explanations must also be considered before that conclusion can be accepted firmly. One possible explanation for the absence of an effect might be a color repetition disadvantage similar to that reported by Fox and de Fockert (2001). They conducted an experiment that focused on possible interfering effects of color by examining whether color repetition caused a disadvantage similar to that caused by location repetition (Fox \& de Fockert, 2001). A color cue was presented for $900 \mathrm{msec}$ at the fixation point. This cue was followed by another item-a neutral attractor, which served to remove attention from the color cue. After $900 \mathrm{msec}$, the target replaced this neutral attractor. Participants detected the target faster when it had a different color than the cue, compared with the situation where cue and target shared the same color. This indicates a disadvantage for color repetition.

A color repetition disadvantage from the cue to the target may have counteracted a positive cuing effect in Experiment 1 , because that experiment was similar in some ways to the experiment of Fox and de Fockert (2001). Critically, both experiments used long displays of a color precue. Although Experiment 1 used an empty screen rather than a neutral attractor in the interval between the cue and the target stimulus, it is still possible that the processes operating to produce a color repetition disadvantage eliminated the cuing effect in this situation.

To cater for this possibility, the second experiment used different stimulus probabilities to create color expectations (Ciaramitaro, Cameron, \& Glimcher, 2001; Geller, 1977). Stimulus probability has been shown to have a strong effect on RT (Fitts, Peterson, \& Wolpe, 1963). For example, Geller demonstrated a stimulus probability effect for color even when it was task irrelevant. He used stimuli defined by three dimensions (location, color, and shape). Each of these dimensions had two possible values - that is, location either "left" or "right" of fixation, the color "red" or "green," and the shape of either an "upwards" or "downwards" pointing triangle. In each condition, participants had to discriminate between the two values of one dimension and their choice RT was measured. The stimulus probabilities for all three dimensions were varied, and Geller evaluated the effect of stimulus probability on the irrelevant dimensions. Irrelevant location probability was most influential and affected color and shape discrimination. More importantly, irrelevant color probability also had an effect on shape discrimination. Thus, these results demonstrate that stimulus probability can create an effect of color expectation even when color information is not required to perform the task.

Manipulating stimulus probability might, therefore, create stronger effects than cuing paradigms. Most importantly for the present concerns, it avoids a possible color repetition effect because there is no precue.

Accordingly, expectations in this experiment were created by varying the probability of the form in question to be displayed in one of two colors. Probabilities were either $80 \%$ or $20 \%$. Identical to Experiment 1, geometrical forms were used as stimuli in a four-choice discrimination task. Again, the discrimination task was independent of the color information, so participants could choose to ignore the color. If participants make use of this probability information, however, then performance should be better in trials where the color expectation is met (i.e., the geometrical form is in the high probability color) than in trials where it is not met. ${ }^{2}$

\section{Method}

Participants. For this experiment, 40 undergraduate students (10 male) at the University of Otago, New Zealand were recruited. They participated to fulfill a first-year course requirement. Their ages ranged from 17 to 36 years. Again, all of them reported having normal color vision.

Stimuli and Procedure. Stimuli were the same four geometrical forms that were employed in the previous experiment. The red color used in the experiment was also the same as before, as was the procedure that was used to determine a matching green color.

Each participant was tested in 1 practice block with 28 trials, followed by 12 experimental blocks with 40 trials each. All aspects of the practice block were exactly like the practice block in Experiment 1 . A single session lasted approximately $45 \mathrm{~min}$.

Again, the participants received new verbal instructions for the experimental blocks. In experimental trials, no cues were presented; instead, forms were presented in either red or green. One group of participants had $80 \%$ red targets and $20 \%$ green targets; the other group had $20 \%$ red targets and $80 \%$ green targets. In the first experimental block, the target was presented for $70 \mathrm{msec}$. Identical to Experiment 1, in the remaining blocks, the presentation time was adjusted according to the accuracy of the participant. Adjustment was similar to that of Experiment 1; the only difference was a general raise in the base level, which determined the amount that presentation time would decrease or increase, from $40 \mathrm{msec}$ to $50 \mathrm{msec}$.

All other aspects not mentioned in this description of the procedure were identical to those described in Experiment 1.

\section{Results and Discussion}

For the same reason as that in Experiment 1, trials with RTs shorter than 200 msec (approximately $0.39 \%$ of all trials) were excluded from the analysis of PC. For analyses of RT, the lower limit was also $200 \mathrm{msec}$, and 2,500 $\mathrm{msec}$ was chosen as an upper limit. Overall, $2.29 \%$ of all trials were excluded because of late responses. Mean accuracies and RTs for the two levels of the color probability factor are displayed in Table 2.

Analysis of accuracy. An ANOVA was conducted with the within-subjects factors of probability (high vs. low) and form (triangle, square, pentagon, hexagon).

Unexpectedly, accuracy was higher in the low probability color condition than in the high probability color condition, but this $2.5 \%$ advantage did not reach significance 
$\left[F(1,39)=1.33, M S_{\mathrm{e}}=368.93, p=.255\right]$. Identical to Experiment 1, there was a main effect of stimulus form $\left[F(3,117)=41.22, M S_{\mathrm{e}}=274.65, p<.001\right]$, with triangles being easier to identify than the other forms.

As for Experiment 1, a 95\% confidence interval was calculated for the true population size of the probability cuing effect, and the results indicated that this effect is likely to be between $-6.83 \%$ and $1.87 \%$. Again, the results indicate that there is no large cuing benefit.

Analysis of RT. As for accuracy, a small advantage for low probability colors over high probability colors was found. Again, the difference was not significant $[F(1,39)=$ $\left.0.81, M S_{\mathrm{e}}=7,011.5, p=.37\right]$. The $95 \%$ confidence interval for RT showed that the true population size of the probability effect was somewhere between $-10.5 \mathrm{msec}$ and $27.4 \mathrm{msec}$. As before, this effect suggests no or only a small probability-based cuing effect. The discrimination advantage for triangles observed in the accuracy data was also present in the RT data, leading to a main effect for stimulus form $\left[F(3,117)=70.58, M S_{\mathrm{e}}=6,149.0, p<.001\right]$. Form also interacted with color probability $[F(3,117)=4.47$, $\left.M S_{\mathrm{e}}=3,477.9, p<.01\right]$. For triangles and hexagons, responses were faster when the color had a high probability than when it had a low probability, whereas for pentagons and squares, responses were slower in the high probability condition than in the low probability condition. The reason for this interaction is unclear.

If color expectation leads to signal enhancement for items in the expected color, performance should be better for the high probability color than for the low probability color. Instead, performance did not differ for these two colors. Accuracy was slightly higher in the low probability condition than in the high probability condition, but this difference was not significant. This result again indicates that advance information about color does not enhance the perceptual signal of the to-be-identified form in this single-item task.

\section{GENERAL DISCUSSION}

The two experiments reported here investigated the mechanisms responsible for previously observed color cuing effects (Vierck \& Miller, 2005). Two potential mechanisms were considered: signal enhancement versus uncertainty reduction. If the color cuing effect found in multiple-item displays was caused by signal enhancement, then advance color information should also lead to a processing advantage in single-item displays, because processing enhancement of to-be-discriminated forms in the expected color would make it easier to perform the task.

Table 2

Average Percentage Correct (PC) and Reaction Time (RT, in Milliseconds) As a Function of Color Probability in Experiment 2

\begin{tabular}{ccc}
\hline & \multicolumn{2}{c}{ Dependent Variable } \\
\cline { 2 - 3 } Trial Condition & PC & RT \\
\hline High probability & 57.9 & 947 \\
Low probability & 60.4 & 938 \\
\hline
\end{tabular}

On the other hand, if uncertainty reduction was responsible for the previous color cuing effects, then no cuing advantage should occur in single-item displays, because there were no distractor items to contribute uncertainty to the display.

Two different techniques were used to create color expectations in these two experiments. In Experiment 1, participants were provided with color precues, whereas in Experiment 2, color probability was varied. The results of both experiments indicate that foreknowledge of the likely stimulus color leads to little or no improvement in form processing in single-item displays.

Because the present conclusions are based on a null effect, we used confidence intervals to compare the present color cuing effect sizes found in single-item displays without distractor items against those found in previous experiments with multiple-item displays containing several distractor items (Vierck \& Miller, 2005, 2006), where color cuing is known to aid selection. We included the data of four previous experiments that were chosen because of their similarities with the experiments reported here. In all of these experiments, (1) colors were easy to differentiate from each other, (2) valid color cues had a high probability of being correct, and (3) valid color cues were compared with neutral color cues. We combined the data of these four previous experiments and calculated a 95\% confidence interval to estimate the color cuing effect with multiple-item displays. For accuracy, the previous data indicate that the population color cuing effect for these displays with distractor items lies somewhere between $4.08 \%$ and $7.2 \%$, and this effect is clearly larger than the maximum possible effect sizes obtained in the present experiments with single-item displays (maximum effects of $1.68 \%$ and $1.87 \%$ for Experiments 1 and 2, respectively). Similarly, for RT, the color cuing effect for displays with distractor items was found to lie between $70.9 \mathrm{msec}$ and $101.2 \mathrm{msec}$, which is substantially larger than the maximum possible effects in the present experiments (38.2 msec and $27.4 \mathrm{msec}$ for Experiments 1 and 2, respectively). Thus, it is clear that advance color information produces little or no facilitation of form discrimination when distractor stimuli are absent, in comparison with when they are present. This result suggests that previous color cuing effects in the presence of distractors resulted primarily or exclusively from uncertainty reduction, with no direct signal enhancement for stimuli in the anticipated color.

These findings extend the results of Moore and Egeth (1998) and Brawn and Snowden (1999) to single-item displays. These authors also explored the possibility of signal enhancement as a mechanism behind observed color cuing effects, but they did so using multiple-item displays. As was outlined in the introduction (cf. Vierck \& Miller, 2005), such displays might not provide the strongest test of color cuing effects. Together with our study, however, the overall results strongly suggest that no direct signal enhancement takes place when advance color information is provided. Rather, it seems that the usefulness of color is restricted to situations in which it helps the selection process to find the right item to process. 
Although our findings reported here are consistent with the idea of uncertainty reduction, Vierck and Miller (2006) reported results, which seem difficult to explain with the uncertainty reduction account. They conducted a variation of the RSVP task that was described previously, but they used two slightly overlapping letters per frame instead of one letter per frame. As before, color cues were provided at the beginning of each trial, and participants had to discriminate whether a target letter was displayed in its upper or lower case version. They found, as before, a clear color cuing benefit, but this benefit was not different in size from the benefit that was observed in the experiment with only a single letter per frame. Thus, an increase in the number of stimuli per frame did not lead to an increase of the validity effect. The uncertainty reduction hypothesis, however, would seem to predict an increase in the validity effect when there are more distractor stimuli, because uncertainty reduction would be more helpful in that case. That is, valid trials would again isolate the target successfully, leading to a cuing benefit. In neutral trials, however, more uncertainty was present than in previous experiments. This should have impaired performance in this condition, which was not the case. Although these findings seem contrary to the predictions of uncertainty reduction, they are not decisive. Signal detection models based on internal noise and the assumption that the choices are made in favor of the stimulus with the maximum response (Eckstein, Thomas, Palmer, \& Shimozaki, 2000) need only predict a very small accuracy change because of uncertainty reduction under the conditions of this experiment. Thus, the hypothesis of uncertainty reduction cannot be ruled out based on the failure to observe this change.

In addition, the present results are in line with Shiu and Pashler's (1994) findings. Those authors reported no signs of signal enhancement in a single-item display when location was cued. But, as has been shown by Luck et al. (1996), this result might have been because of the use of a very short SOA that might not have allowed enough time for signal enhancement to take place. In the present Experiment 1 , the SOA was very long - much longer than the one employed by Luck and colleagues or Shiu and Pashler. Consequently, a lack of preparation time can be ruled out as the reason for our nil validity effect, strengthening the conclusion that signal enhancement is not the mechanism responsible for the observed color cuing effect in multiple-item displays (Vierck \& Miller, 2005).

Our findings are in contrast to results of other researchers who have reported a benefit in location precuing paradigms in single-item displays, and therefore support signal enhancement (e.g., Bashinski \& Bacharach, 1980; Henderson, 1991, 1996; Van der Heijden et al., 1985). For example, Bashinski and Bacharach's design was very similar to the one used here. In both studies, the task could be fulfilled without the advance information (color or location), and no other competing stimuli were present. Despite that, those authors found a clear sensitivity increase for the valid location condition, which has been interpreted as a sign of perceptual tuning for stimuli at attended locations in early visual processing (Bashinski \&
Bacharach, 1980). In our design, participants had the analogous opportunity to allocate attention to the cued color channel. If a cuing effect could be found with a single stimulus for location cuing, this should also be possible for color cuing if signal enhancement occurs. The fact that we failed to find an advantage of advance color information indicates differences in the processing of location and color. It may be that location processing is generally more effective. Alternatively, the mechanisms themselves may differ. Together, these and other results indicate that for location cuing, both signal enhancement and uncertainty reduction may operate (see, e.g., Luck et al., 1996; Shiu \& Pashler 1994). In contrast, for color cuing, only evidence for uncertainty reduction but not for signal enhancement has been found so far. Thus, the underlying mechanisms of location and color cuing may well differ.

Location has frequently been regarded as special and more important than other features in attentional selection (e.g., Tsal \& Lavie, 1988, 1993). Tsal and Lavie (1993), for example, demonstrated location dominance in a selection task. They asked participants to identify a letter in one of two locations. In each trial, a small dot next to each of these possible locations was presented. One of the dots was colored, and the color defined which location the target letter was in. The location of the dot itself was irrelevant; only the color mattered. The results showed that RT was faster when the colored dot was next to the location of the target than when the colored dot was on the opposite side. The authors concluded that location is more important than other features in selection. Similar results have been reported repeatedly (Cave \& Pashler, 1995; Garner, 1987; Kim \& Cave, 2001; Shih \& Sperling, 1996; Tsal \& Lavie, 1988). It might be that this special status of location is because of a difference in mechanisms between location and other features. Location benefits seem to be caused by signal enhancement and uncertainty reduction, whereas other features seem to only help to reduce uncertainty. This difference between location cuing and cuing of other features points toward a more fundamental role of location information in attentional selection. Our findings are consistent with this possibility, because they suggest that advance color information reduces uncertainty for selection processes, but does not result in signal enhancement.

\section{AUTHOR NOTE}

The experiments reported here were conducted in partial fulfillment of requirements for E.V.'s doctoral degree from the University of Otago. We thank three anonymous reviewers for helpful comments. This research was supported in part by a Marsden grant from the Royal Society of New Zealand to J.M. Correspondence concerning this article should be addressed to either E. Vierck or J. Miller at the Department of Psychology, University of Otago, Dunedin, New Zealand (e-mail: esther@ psy.otago.ac.nz or miller@psy.otago.ac.nz).

\section{REFERENCES}

Bashinski, H. S., \& BaCharach, V. R. (1980). Enhancement of perceptual sensitivity as the result of selectively attending to spatial locations. Perception \& Psychophysics, 28, 241-248.

Brawn, P., \& SNOWden, R. J. (1999). Can one pay attention to a particular color? Perception \& Psychophysics, 61, 860-873.

Cave, K. R., \& Pashler, H. (1995). Visual selection mediated by loca- 
tion: Selecting successive visual objects. Perception \& Psychophysics, 57, 421-432.

Cheal, M., \& Gregory, M. (1997). Evidence of limited capacity and noise reduction with single-element displays in the location-cuing paradigm. Journal of Experimental Psychology: Human Perception \& Performance, 23, 51-71.

Ciaramitaro, V. M., Cameron, E. L., \& Glimcher, P. W. (2001). Stimulus probability directs spatial attention: An enhancement of sensitivity in humans and monkeys. Vision Research, 41, 57-75.

Eckstein, M. P., Thomas, J. P., Palmer, J., \& Shimozaki, S. S. (2000). A signal detection model predicts the effects of set size on visual search accuracy for feature, conjunction, triple conjunction, and disjunction displays. Perception \& Psychophysics, 62, 425-451.

Fitts, P. M., Peterson, J. R., \& Wolpe, G. (1963). Cognitive aspects of information processing: II. Adjustments to stimulus redundancy. Journal of Experimental Psychology, 65, 423-432.

Fox, E., \& DE FOCKERT, J.-W. (2001). Inhibitory effects of repeating color and shape: Inhibition of return or repetition blindness? Journal of Experimental Psychology: Human Perception \& Performance, 27, 798-812.

GaRnER, W. R. (1987). Location and color as cuing dimensions in contingent classification. Perception \& Psychophysics, 41, 202-210.

Geller, E. S. (1977). Latencies to name one of three stimulus dimensions: A study of probability effects and dimension integrality. Perception \& Psychophysics, 22, 70-76.

GrindLey, G. C., \& Townsend, V. (1968). Voluntary attention in peripheral vision and its effects on acuity and differential thresholds. Quarterly Journal of Experimental Psychology, 20, 11-19.

Henderson, J. M. (1991). Stimulus discrimination following covert attentional orienting to an exogenous cue. Journal of Experimental Psychology: Human Perception \& Performance, 17, 91-106.

Henderson, J. M. (1996). Spatial precues affect target discrimination in the absence of visual noise. Journal of Experimental Psychology: Human Perception \& Performance, 22, 780-787.

HuYnH, H. (1978). Some approximate tests for repeated measurement designs. Psychometrika, 43, 161-175.

KIM, M.-S., \& CAVE, K. R. (2001). Perceptual grouping via spatial selection in a focused-attention task. Vision Research, 41, 611-624.

Kinchla, R. A., Chen, Z., \& Evert, D. (1995). Precue effects in visual search: Data or resource limited? Perception \& Psychophysics, 57, 441-450.

Kornblum, S. (1969). Sequential dependencies as a determinant of choice reaction time: A summary. Acta Psychologica, 30, 54-55.

Lu, Z.-L., \& Dosher, B. A. (2000). Spatial attention: Different mechanisms for central and peripheral temporal precues? Journal of Experimental Psychology: Human Perception \& Performance, 26, 1534-1548.

Luck, S. J., Hillyard, S. A., Mouloua, M., \& Hawkins, H. L. (1996). Mechanisms of visual-spatial attention: Resource allocation or uncertainty reduction? Journal of Experimental Psychology: Human Perception \& Performance, 22, 725-737.

McCann, R. S., Folk, C. L., \& Johnston, J. C. (1992). The role of spatial attention in visual word processing. Journal of Experimental Psychology: Human Perception \& Performance, 18, 1015-1029.

Moore, C. M., \& Egeth, H. (1998). How does feature-based attention affect visual processing? Journal of Experimental Psychology: Human Perception \& Performance, 24, 1296-1310.

Palmer, J., Ames, C. T., \& Lindsey, D. T. (1993). Measuring the effect of attention on simple visual search. Journal of Experimental Psychology: Human Perception \& Performance, 19, 108-130.
Posner, M. I., Snyder, C. R. R., \& Davidson, B. J. (1980). Attention and the detection of signals. Journal of Experimental Psychology: General, 109, 160-174.

SHIH, S.-I, \& SPERLING, G. (1996). Is there feature-based attentional selection in visual search? Journal of Experimental Psychology: Human Perception \& Performance, 22, 758-779.

Shiu, L.-P., \& PASHLER, H. (1994). Negligible effect of spatial precuing on identification of single digits. Journal of Experimental Psychology: Human Perception \& Performance, 20, 1037-1054.

SPITCZOK VON BRISINSKI, I. (1994). Ultrafast display buildup with standard VGA on MS-DOS computers. Behavior Research Methods, Instruments, \& Computers, 26, 335-336.

TheEuwes, J. (1989). Effects of location and form cuing on the allocation of attention in the visual field. Acta Psychologica, 72, 177-192.

TsaL, Y., \& LAMY, D. (2000). Attending to an object's color entails attending to its location: Support for location-special views of visual attention. Perception \& Psychophysics, 62, 960-968.

Tsal, Y., \& LaVIE, N. (1988). Attending to color and shape: The special role of location in selective visual processing. Perception \& Psychophysics, 44, 15-21.

TSAL, Y., \& LaVIE, N. (1993). Location dominance in attending to color and shape. Journal of Experimental Psychology: Human Perception \& Performance, 19, 131-139.

Van der Heijden, A. H., Schreuder, R., \& Wolters, G. (1985). Enhancing single-item recognition accuracy by cueing spatial locations in vision. Quarterly Journal of Experimental Psychology, 37A, 427-434.

Vierck, E., \& Miller, J. (2005). Direct selection by color for visual encoding. Perception \& Psychophysics, 67, 483-494.

VierCK, E., \& Miller, J. (2006). Effects of task factors on selection by color in the rapid serial visual presentation (RSVP) task. Perception \& Psychophysics, 68, 1324-1337.

\section{NOTES}

1. An additional analysis was conducted that separated between trials in which the cue color was identified correctly and incorrectly at the end of the trial, because participants without knowledge of the cue color are unlikely to have used it for the preceding discrimination task. The average accuracy concerning the question about the color of the precue was $93.8 \%$. The results confirm the findings of the main analysis. Performance in valid trials was not significantly different from performance in invalid trials - neither for accuracy nor for RT-when only trials in which the cue was correctly identified were taken into consideration.

2. With the current probability manipulation, the stimulus in each trial was not only more likely to appear in the high probability color, it was also more likely to follow a stimulus of the high probability (i.e., from the preceding trial; cf. Kornblum, 1969). If there were a color repetition disadvantage under these circumstances, it could selectively interfere with performance in trials with stimuli in the high probability color, reducing the power of the experiment to detect an advantage for stimuli in this color. This possible confound is not a worry, however, because each colored form was displayed only briefly — on average, $46.2 \mathrm{msec}$. Fox and de Fockert (2001) showed that initial presentations of $50 \mathrm{msec}$ are not long enough to result in a color repetition disadvantage for processing of subsequent stimuli.

(Manuscript received October 25, 2005; revision accepted for publication August 30, 2006.) 